\title{
Metal piezoelectric semiconductor field effect transistors for piezoelectric strain sensors
}

\author{
Yuh-Renn Wu and Jasprit Singh ${ }^{\text {a) }}$ \\ Department of Electrical Engineering and Computer Science, University of Michigan, Ann Arbor, \\ Michigan 48109
}

(Received 15 September 2003; accepted 17 June 2004)

\begin{abstract}
In this letter, we examine the potential of a functional device that can have good transistor and stress sensor properties. The device examined is based on the use of a thin oxide with high piezoelectric coefficients under the gate region. Channel charge and current are controlled by gate voltage or by stress. We examine the performance of two classes of heterostructures that are important semiconductor technologies: (i) $\mathrm{Si} / \mathrm{SiO}_{2} / \mathrm{BaTiO}_{3}$ heterostructure junctions that would be an important breakthrough for silicon sensor technology and (ii) $\mathrm{GaN} / \mathrm{AlN} / \mathrm{BaTiO}_{3}$ heterostructure field effect transistors. The calculations show that with a very thin piezoelectric layer we can have a highly sensitive stress sensor and transistor. For optimum performance, the piezoelectric layer thickness should be 30-60 А. (C) 2004 American Institute of Physics. [DOI: 10.1063/1.1784039]
\end{abstract}

In recent years, devices exploiting piezoelectric material have been developed for electromechanical actuators and sensors. ${ }^{1}$ In general, piezoelectric materials are used as piezoresistive sensors. While applying bias current on the piezoresistive material, the variation of resistance induced by strain effect leads to the voltage drop. The voltage signal is then fed to the gate of field effect transistors (FETs) with high input impedance and then transduced to current by the FET. This allows us to estimate the strain by measuring the current. However, two or more devices including capacitors are needed in these detecting systems to amplify the signal, which limits the operation frequency and increases the power consumption. Furthermore, the strain measured by the piezoresistive materials is the average value cross the resistors, which is not suitable for small size detector. These considerations limit applications to microelectromechanical system.

Recently, piezoceramics grown on silicon or nitride based heterostructure junctions have attracted considerable interest. $^{2-6}$ For experimentalists who are working in this area, several important questions remain unanswered: (i) What are some critical physical properties that need to be measured? (ii) What kinds of devices can show performance that is superior to the existing devices? (iii) Can polar oxidesemiconductor structures create physical effects that are not possible in existing technologies? It is known that there is a strong fixed polarization charge at the heterointerface, which introduces very large electric fields and band bending, and induces a two-dimensional electron gas (2DEG) at the semiconductor heterostructure interface. For the most part, these studies have focused on the 2DEG induced by the static built in strain in the heterostructure. However, it is important to examine the response of the 2DEG to dynamic strain variations. As we know, dynamic strain leads to the change of piezoelectric polarization, which would affect the band bending and the 2DEG density. In field effect transistors (FETs), once the strain is directly applied in the FET, the variation of the 2DEG caused by strain will affect the drain-source current. Therefore we can directly measure strain through variation of drain-source current. Such a device design will have

\footnotetext{
${ }^{a)}$ Electronic mail: yrw@umich.edu
}

several advantages. First, these sensors are directly embedded to FETs without the need of $R C$ circuit, and therefore the frequency limitation would be much lower than the traditional device. The size of the FET can be submicrometer, so that the size of sensor can be extremely small. Such a power saving higher frequency FET sensor would have a great impact on sensor technology.

In this letter, we will present this theoretical model for two classes of junctions that are important for device technologies: (i) silicon based $\mathrm{Si} / \mathrm{SiO}_{2} / \mathrm{BaTiO}_{3}$ junctions and (ii) $\mathrm{GaN}$ based $\mathrm{GaN} / \mathrm{AlN} / \mathrm{BaTiO}_{3}$ junctions. Owing to their wide band-gap, the nitride based materials are especially important for the high temperature and high voltage application. The ferroelectric material, $\mathrm{BaTiO}_{3}$, is selected due to its high piezoelectric constant ${ }^{7,8}$ which is more sensitive to the variation of strain.

The formalism developed is generic and can be applied to other "smart" oxides as well. The basic structure examined by us is shown schematically in Fig. 1(a). As shown, a

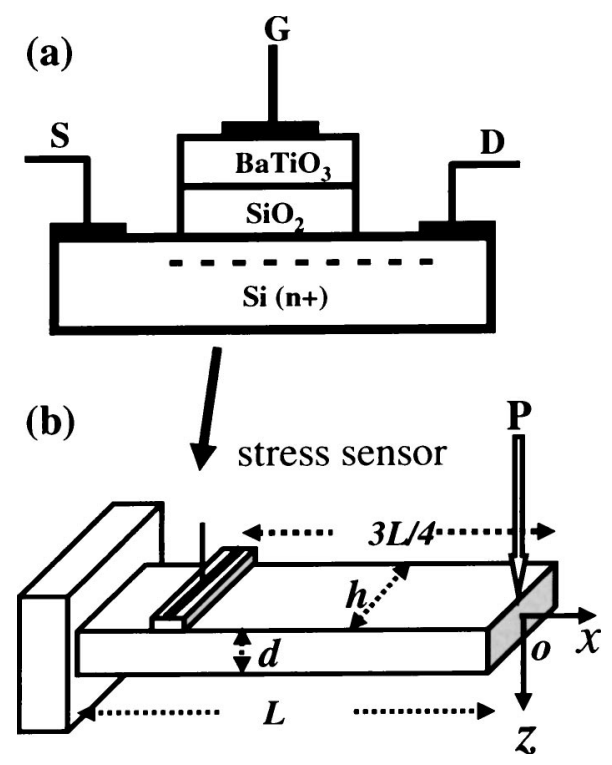

FIG. 1. (a) A schematic of the MPISFETs structure. (b) The MPISFETs is grown on top of the cantilever. 
"smart oxide" (in our simulation, this is $\mathrm{BaTiO}_{3}$ ) is placed between the gate and 2D channel of a FET. We note that since at present oxides such as $\mathrm{BaTiO}_{3}$ are likely to have a large defect density and very poor mobility, we design the smart FET so that the free carrier density is essentially at the high quality $\mathrm{Si} / \mathrm{SiO}_{2}$ interface. The metal-piezoelectricinsulator semiconductor field effect transistor (MPISFET) sensor is assumed to be grown on silicon cantilever as shown in Fig. 1(b). When a force $P$ applied on the side of the beam, the deflection $\delta z$ in the $z$ direction is given by

$$
\delta z=\frac{L^{3}}{3 E I} P,
$$

where $E$ is Young's modulus of the beam, $I$ is the momentum of inertia, and $L$ is the length of the beam. The momentum of inertia $I$ of a rectangular beam is $h d^{3} / 12$, where $h$ is the beam width and $d$ is the beam height. When the cantilever is under pressure, the stress $\sigma_{x}$ is $\epsilon_{x} E$, where $\epsilon_{x}$ is the strain and given by

$$
\epsilon_{x}(x,-d / 2)=\frac{\sigma_{x}(x, z)}{E}=\frac{3 x(d / 2) \delta z}{L^{3}},
$$

where $z=-d / 2$ since the MPISFET is grown on top of the beam. We can also derive the strain $\epsilon_{y}$ is equal to $\tau \epsilon_{x}$, where $\tau$ is the Poisson ratio. The detailed formalism can be found in Ref. 9. Here we have assumed that MPISFETs are much thinner than the height of the beam, and therefore the strain, $\epsilon_{x}$ and $\epsilon_{y}$, on top of beam is equal to the strain on the piezoelectric material. For tetragonal materials such as $\mathrm{BaTiO}_{3}{ }^{7}$ it can be shown that the polarization in the $z$ direction $P_{z}$ is given by

$$
P_{z}=P_{\mathrm{sp}}+\left(e_{13}-e_{33} \frac{C_{13}}{C_{33}}\right)\left(\epsilon_{x}+\epsilon_{y}\right),
$$

where $e_{i j}$ is the piezoelectric constant, $C_{i j}$ is the elastic constant, and $P_{\mathrm{sp}}$ is the spontaneous polarization of the piezoceramics, which is $-0.26 \mathrm{C} / \mathrm{m}^{2}$ for $\mathrm{BaTiO}_{3}$. The values of $C_{13}$ and $C_{33}$ for $\mathrm{BaTiO}_{3}$ are 211 and $160 \mathrm{GPa}$, respectively, and $e_{31}$ and $e_{33}$ of $\mathrm{BaTiO}_{3}$ are -3.88 and $5.48 \mathrm{C} / \mathrm{m}^{2}$, respectively. The dielectric constant of $\mathrm{BaTiO}_{3}$ is $48 \varepsilon_{0}{ }^{7}$. Once the polarization $P_{z}$ is obtained, the two-dimensional electron gas induced by the polarization can be calculated by a chargecontrol model, ${ }^{10,11}$ which solves the Poisson equation and Schrödinger equation self-consistently.

To study the performance of the MPISFET sensor, we first begin with $\mathrm{Si} / \mathrm{SiO}_{2} / \mathrm{BaTiO}_{3}$ heterostructure junctions. Traditionally, silicon is used for mechanical sensors, because it combines well-established electronic properties with excellent mechanical properties. ${ }^{12}$ Therefore, the component of the catilever is assumed to be silicon in this case. The Young's modulus and Poisson ratio for silicon is $130 \mathrm{Gpa}$ and 0.28 , respectively. ${ }^{13}$ In Fig. 2 we show how the sheet charge density in the channel changes as stress increases. In Fig. 2(a), we show the 2DEG at the $\mathrm{BaTiO}_{3} / \mathrm{SiO}_{2}$ junction while in Fig. 2(b), it is shown at the $\mathrm{Si} / \mathrm{SiO}_{2}$ junction. We see that for a 50 and $30 \AA \mathrm{BaTiO}_{3}$ film, a large part of the electron free charge resides at the $\mathrm{BaTiO}_{3} / \mathrm{SiO}_{2}$ interface. Since it is expected that mobile charge will have very poor transport properties and may cause deleterious trap related problems, it is important that most of the electron charge resides at the $\mathrm{Si} / \mathrm{SiO}_{2}$ interface. We see that once $\mathrm{BaTiO}$ thickness reaches $20 \AA$, the channel charge is all at the $\mathrm{Si} / \mathrm{SiO}_{2}$ inter-

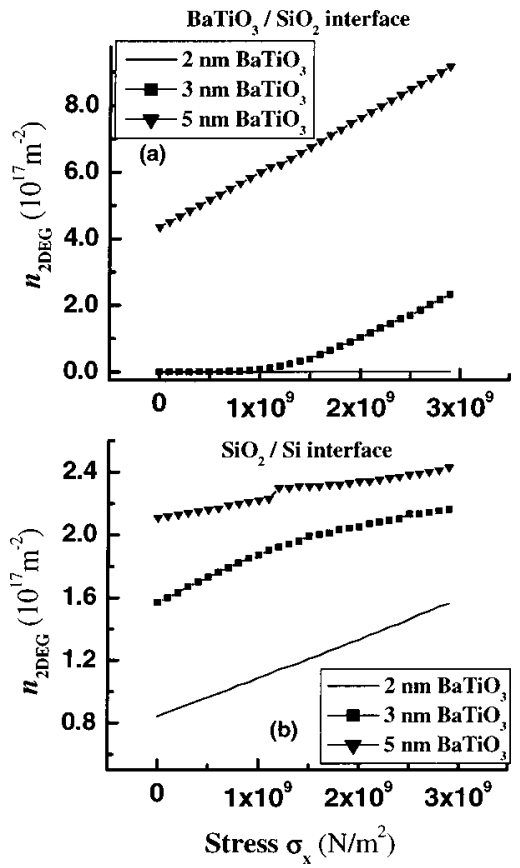

FIG. 2. Calculated sheet charge densities, $\left(n_{2 \mathrm{DEG}}\right)$, for the $\mathrm{Si} / \mathrm{SiO}_{2} / \mathrm{BaTiO}_{3}$ heterostructure junctions. (a) The $n_{2 \mathrm{DEG}}$ at the $\mathrm{SiO}_{2} / \mathrm{BaTiO}_{3}$ interface. (b) The $n_{2 \mathrm{DEG}}$ at the $\mathrm{Si} / \mathrm{SiO}_{2}$ interfaces.

face and the device acts as a strained metal-oxidesemiconductor FET but with a very large stress response. We find a slope of $d n / d \sigma_{x}=2.57 \times 10^{7} \mathrm{~N}^{-1}$ for the optimal configuration. As can be seen form Fig. 2(b), a large change in channel current is expected as stress changes.

We next consider $\mathrm{GaN} / \mathrm{AlN} / \mathrm{BaTiO}_{3}$ heterojunctions. It is known there is a large spontaneous polarization in the nitrides. Additionally, the piezoelectric effect is also very strong, which would induce large polarization under the strain condition. Therefore, nitrides would be good choice for piezoelectric sensors. The crystal structure of nitride is wurtzite. The piezoelectric polarization induced by the strain would be the same as Eq. (3). The material of cantilever is assumed to be $\mathrm{GaN}$ in this calculation. The elastic constants $C_{13}$ of $\mathrm{AlN}$ and $\mathrm{GaN}$ are 100 and $110 \mathrm{GPa}$, respectively, and the $C_{33}$ of both are $390 \mathrm{GPa}$. The piezoelectric constants $e_{31}$ of AlN and $\mathrm{GaN}$ are -0.58 and $-0.34 \mathrm{C} / \mathrm{m}^{2}$, respectively. The $e_{33}$ of $\mathrm{AlN}$ and $\mathrm{GaN}$ are 1.55 and $0.67 \mathrm{C} / \mathrm{m}^{2}$, respectively. The piezoelectric constants of GaN and AlN are similar, which cancels part of the fixed polar charge variation at the GaN/AIN interface induced by the strain. Therefore, a structure with a thin $\mathrm{BaTiO}_{3}$ layer on top is considered to improve the sensor properties. Figure 3 shows a schematic of the structure and the results for using $\mathrm{GaN} / \mathrm{AlN} / \mathrm{BaTiO}_{3}$ heterostructure junctions. Once again a large thickness of $\mathrm{BaTiO}_{3}$ layer leads to higher band bending in the $\mathrm{BaTiO}_{3}$ layer and accumulation of sheet charge density at the $\mathrm{AlN} / \mathrm{BaTiO}_{3}$ interface. This would lower the performance of sensor and mobility of FETs channel. The optimal thickness of $\mathrm{BaTiO}_{3}$ is $30 \AA$, for which the slope $d n / d \sigma_{x}$ is equal to $1.608 \times 10^{7}\left(\mathrm{~N}^{-1}\right)$.

The parameters for oxides used in the calculations are assumed to be those for perfect bulk materials, However, in reality, the properties of thin film piezoelectric or pyroelectric material may have difference from the bulk crystal. Recently, several theoretical and experimental reports ${ }^{14,15}$ indicate that the spontaneous polarization of $\mathrm{BaTiO}_{3}$ would 


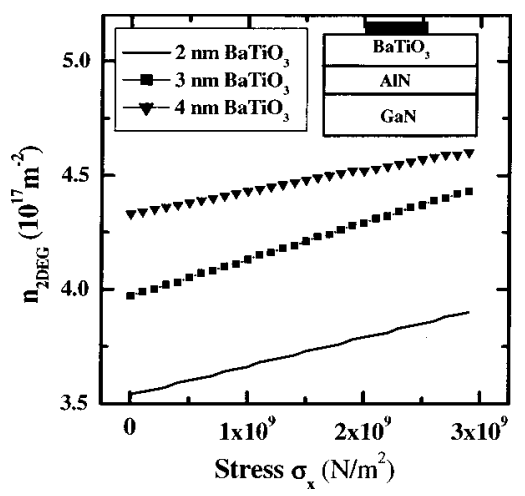

FIG. 3. Calculated sheet charge densities, $\left(n_{2 \mathrm{DEG}}\right)$, for the GaN/AlN heterostructure interface. The thickness of AlN layer is $30 \AA$.

decrease when the film thickness is smaller than $100 \AA$. There are reports of a critical thickness, $24 \AA$ for $\mathrm{BaTiO}_{3}$ thin layer to keep its spontaneous polarization. The spontaneous polarization decrease to $-0.05 \mathrm{C} / \mathrm{m}^{2}$ at the critical thickness. In order to determine the influence caused by parameter changes, we examine the optimal thickness for different dielectric constant and spontaneous polarization. Figure 4 shows the calculated optimal $\mathrm{BaTiO}_{3}$ layer thickness versus the spontaneous polarization for different dielectric constants. When the spontaneous polarization decreases to $-0.05 \mathrm{C} / \mathrm{m}^{2}$, the optimum $\mathrm{BaTiO}_{3}$ changes form 30 to $60 \AA$. It is also clear that optimal polar material layer thickness is roughly proportional to $1 / \varepsilon$. We can see in Fig. 4 that when $\varepsilon$ is assumed to be $200 \varepsilon_{0}$, we obtain an optimum layer thickness of $\sim 85 \AA$. Thus if $\epsilon$ increases or spontaneous polarization decreases, the optimum thickness of polar materials in-

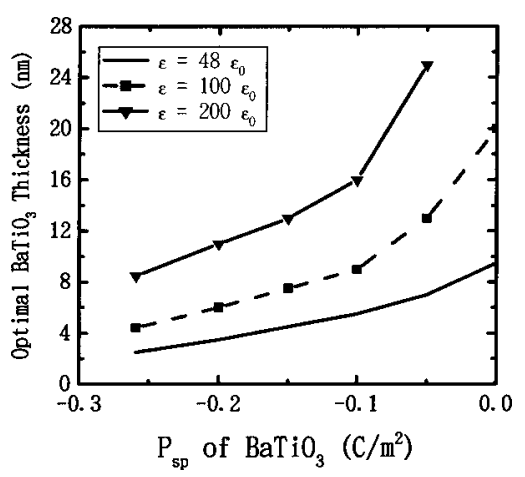

FIG. 4. Calculated optimal $\mathrm{BaTiO}_{3}$ layer thickness of $\mathrm{Si} / \mathrm{SiO}_{2} / \mathrm{BaTiO}_{3}$ heterostructure sensor-FETs vs spontaneous polarization for different dielectric constants. The thickness of $\mathrm{SiO}_{2}$ layer is $8 \AA$. As discussed in the text, at the optimal thickness the 2DEG is located at the $\mathrm{SiO}_{2} / \mathrm{Si}$ interface. creases. In some references, ${ }^{9}$ the $\varepsilon$ is $1900 \varepsilon_{0}$, we obtain an optimum layer thickness of $\sim 800 \AA$. The parameter study of Fig. 4 would be useful for device design.

One must note that the use of polar oxides can also influence tunneling related to the gate current. The band bending will lead to higher tunneling current. ${ }^{16}$ Therefore, an insulator or wide band gap $\left(\mathrm{SiO}_{2}\right.$ and $\left.\mathrm{AlN}\right)$ between polar oxide and semiconductor would be necessary to stop the tunneling effect. Another aspect that needs to be further considered is the electrostatics at the heterostructure interface. Recently, some studies ${ }^{17}$ indicate the band offset at oxide /Si interface can be changed. Therefore, the tunneling mechanism and optimal thickness might be affected. These effects need more experimental data to examine.

In summary, our results show that a very thin piezoelectric layer can allow a super sensitive stress sensor and a high transconductance. Besides, the size of FET sensors can be very small and thin which can be very easily integrated into electronic circuits and microprocessors. An important outcome of our study is that the thickness of the $\mathrm{BaTiO}_{3}$ film is quite small (i.e., in the range of $20-50 \AA$ ). As a result, epitaxial growth technologies such as MBE are needed.

This work was supported by Grant Nos. F001681 and F004815 from the U.S. Office of Naval Research.

${ }^{1}$ A. J. Moulson and J. M. Herbert, Electroceramics 2nd ed. (Wiley, London, 2003).

${ }^{2}$ Y.-Y. Lin and J. Singh, J. Appl. Phys. 91, 9297 (2002).

${ }^{3}$ B. T. Liu, K. Maki, Y. So, V. Nagarajan, R. Ramesh, J. Lettieri, J. H. Haeni, D. G. Schlom, W. Tian, X. Q. Pan, F. J. Walker, and R. A. McKee, Appl. Phys. Lett. 80, 4801 (2002).

${ }^{4}$ B. Shen, W. P. Li, T. Someya, Z. xia Bi, J. Liu., H.-M. Zhou, R. Zhang, F. Yan, Y. Shi, Z.-G. Liu, Y.-D. Zheng, and Y. Arakawa, Jpn. J. Appl. Phys., Part 1 41, 2528 (2002).

${ }^{5}$ A. Schmehl, F. Lichtenberg, H. Bielefeldt, J. Mannhart, and D. G. Schlom, Appl. Phys. Lett. 823077 (2003).

${ }^{6}$ A. P. Dmitriev, V. Y. Kachorovskii, M. S. Shur, and R. Gaska, J. Appl. Phys. 94, 566 (2003).

${ }^{7}$ Z. Li, S.-K. Chan, M. H. Grimsditch, and E. S. Zouboulis, J. Appl. Phys. 70, 7327 (1991).

${ }^{8}$ S. Saha and T. P. Sinha, Phys. Rev. B 62, 8828 (2000).

${ }^{9}$ A. J. Moulson and J. M. Herbert, in Ref. 1, Chap. 6 pp. 381-402.

${ }^{10}$ M. Singh, J. Singh, and U. Mishra, J. Appl. Phys. 91, 2989 (2002).

${ }^{11}$ M. Singh, Y. Zhang, J. Singh, and U. Mishra, Appl. Phys. Lett. 77, 1867 (2000).

${ }^{12}$ K. E. Peterson, Proc. IEEE 70, 420 (1992).

${ }^{13}$ E. Anastassakis and M. Siakavellas, J. Appl. Phys. 90, 144 (2001).

${ }^{14}$ J. Junquera and P. Ghosez, Nature (London) 422, 506 (2003).

${ }^{15}$ M. G. Stachiotti, Appl. Phys. Lett. 84, 251 (2004).

${ }^{16}$ Y.-R. Wu, M. Singh, and J. Singh, J. Appl. Phys. 94, 5826 (2003).

${ }^{17}$ R. A. Mckee, F. J. Walker, B. Nardelli, W. A. Shelton, and G. M. Stocks, Science 300, 1726 (2003). 